\title{
Classification of Objects for Conceptual Design: An Ontology Based Approach
}

\author{
Javier Flores \\ Department of Aircraft Design \\ Samara University \\ Samara, Russia \\ flores.javier.1989@yandex.ru
}

\author{
Nikolay Borgest \\ Department of Aircraft Design \\ Samara University \\ Samara, Russia \\ borgest@yandex.ru
}

\begin{abstract}
The use of Ontologies in the present is becoming very common and widely used in different education areas, some areas of the engineering such as aerospace, can improve with the application of this informatics tools. Classification of aircraft as an object and al his components has become an important research area for the statistics analysis previous of designing a new concept, in this document we are going to explain, how Fluent Editor can help the designer to improve the classification and information analysis used to choose the statistical characteristics of the new concept. On this paper is showed an ontology of aircraft engines. Databases are created for storing, processing, performing calculations, sorting, sampling and presentation of data arrays according to various criteria. Then the created array of data on aircraft engines can be further used as a basis for selecting the engine according to the technical task.
\end{abstract}

Keywords - design, ontology, education, aircraft, engine Introduction

\section{INTRODUCTION}

Design of new technical systems is one of the most challenging kind of engineering activities. The main feature of the conceptual design is the necessity of making decisions without sufficient data or, contrary, with redundant data. It has big difference from "school" tasks, which have the number of inputs that is exactly enough to obtain one certain result. Drawing on the previous experience in the form of prototype statistics plays an important role in conceptual design.

It is known that the key element of the process of creating an airplane is its project, that is, the development of the project both in manual mode and in automated mode. To develop a modern airplane project means to develop a complete set of design and technological documentation both for paper technology and for machine carriers, which makes it possible to create an aircraft in metal and to operate it.

It is clear that modern designs of aircraft and other complex engineering techniques cannot be created without the use of automation systems, so the degree of automation of design processes is largely determined not so much by the capabilities of modern CAD, as by the ability to formalize a particular design task, that is, the designer's ability to give a fairly strict formulation design tasks and a clear completed algorithm for solving it, using the maximum information about a typical design task, then is the use of information about the projected product (airplane).

After determining the design goal, the designer, basing on information, as well as creativity, forms the main idea, that is, the concept of the future aircraft, outlining possible options (alternatives) for the solution of the design task using a design automation system adapted to the specific project task.

Classically, the technical documentation is only the end result of a complex and long process of design work by the creators of the aircraft, aimed at developing a project of a previously non-existent object (aircraft), system and process.

Classification of digitized documents nowadays gains a higher significance due to the rapid growth of digital content. With respect to the growth, organizing them is a big challenge for efficient retrieval information. Therefore, finding and improving solutions for text classification has considerable importance [1].

\section{APPROACH TO CREATE AN ONTOLOGY}

Semantic technologies based on ontologies of the surrounding world, using the connections, rules, logic and conditions described in the ontology. In turn, ontological analysis, in addition to streamlining knowledge of the subject area, also contributes to the quality of the work performed. That is why ontologies are used in the creation of information systems dealing with the activities of both design enterprises and industrial enterprises.

There are two alternative approaches to creating and researching ontologies. The first (formal) approach is based on logic (first-order predicate, descriptive, modal). The second one (linguistic) is based on the study of natural language (semantic in particular) and on the ontology development in large text arrays [2].

The natural language description is the main difference between Fluent Editor and other ontological editors and allows you to master the creation of ontologies for a much wider group of users. The built-in reasoning engine (English Embedded Reasoner) in Fluent Editor automatically generates XML, RDF and OWL files. OWL (English Web Ontology Language) is a language for describing ontologies for the semantic web. The OWL language allows you to describe 
classes and relationships between them, inherent in web documents and applications [3].

Fluent Editor contains an intelligent editor (English Predictive Editor), an editor that independently monitors grammatically and morphologically incorrect text, actively helping the user in the correct writing of ontology [4].

\section{DATABASE GENERATION}

The aircraft, like any other technical object of engineering, is an object of design and represents a complex technical system with a developed hierarchical structure.

In turn, the aircraft is considered as the initial (basic) subsystem, where it is possible to distinguish such subsystems as the glider level, such as the glider, power plants, equipment, equipment, avionics, chassis system, etc. Each of these subsystems under the design is decomposed, that is, it is divided into a number of even smaller subsystems (components), an example in figure 1.

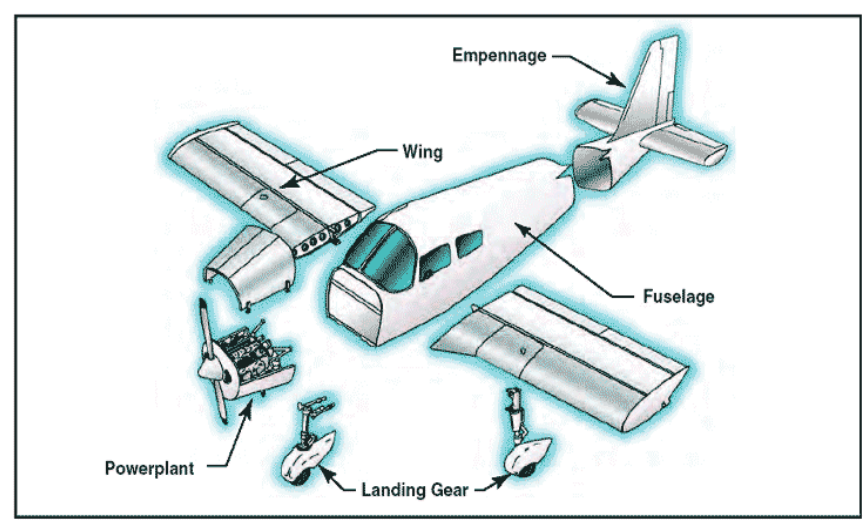

Fig.1. Example of components of the system aircraft

Hierarchical levels are related to each other, this characterizes the structure of the system and regulates the composition of its elements, blocks, aggregates and the relationship of the constituent structures to each other. At the same time, any structure is created to perform certain functions (useful actions, states or properties). For example, the Engine performs the functions of "powering the airplane", "consuming fuel", etc.

Thus, all the elements of the subsystems and their individual elements over the hierarchy levels are related to each other by functional relationships.

Each hierarchical level corresponds to its list of tasks, the solution of which is necessary for making design decisions corresponding to this level, and even more so in the conditions of the CAD functioning when creating the aircraft design. Therefore, in the automated design of aircraft design, an important aspect from the point of view of formalization is its hierarchical structure and the multi-stage design that follows from it [5].

Fig. 2 shows CNL Editor window is the main part of Fluent Editor where you can actually view and edit ontology files. It shows all the CNL phrases, both from the edited file and from the reference ontologies that correspond to every OWL statement. You can click on any phrase from the edited OWL file to modify it, or can also add new phrases [3].

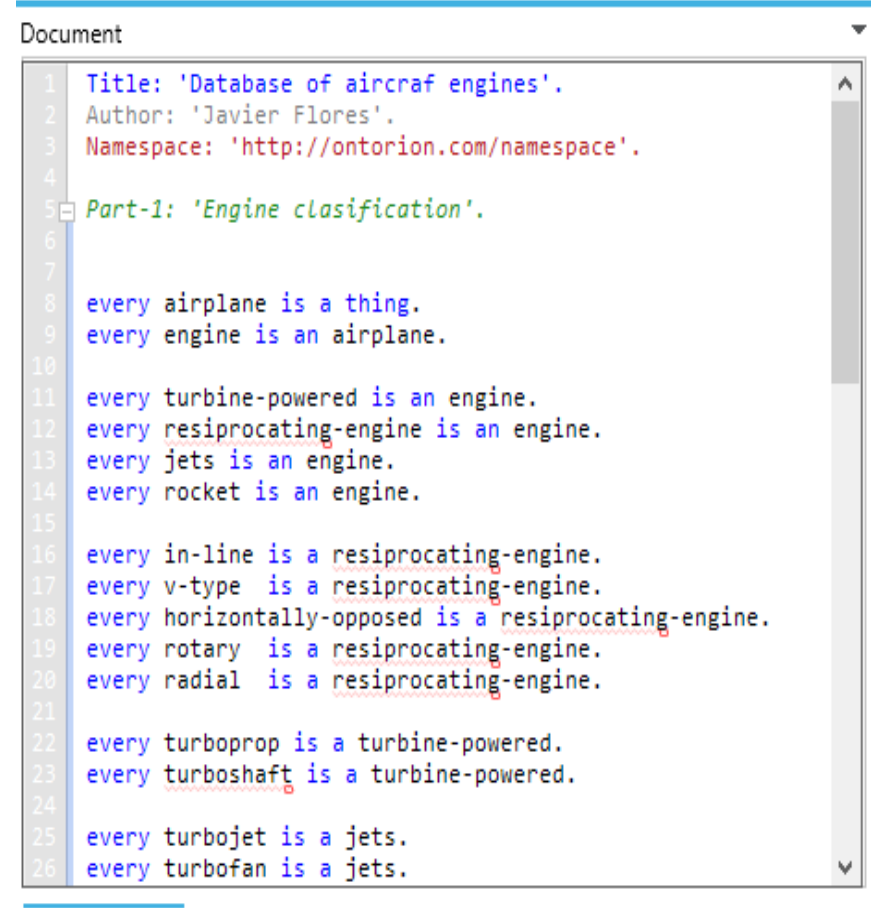

曰Document 品 Document Diagram

Fig. 2. A fragment of the document

\section{TAXONOMY TREE}

Fluent Editor shows in a way of Taxonomy Tree in for of hierarchical levels and how each of the elements are related to each other, this structure facilitates the visualization of the Database. Taxonomy tree is displayed for each OWL file being edited and is built upon data from this OWL file and all included ontologies. Selecting element on the Taxonomy tree will filter all expressions in CNL Editor window to those, which are related explicitly to the selected phrase [2]. The figure 3 shows the Taxonomy tree that helps to navigate and to search systems or subsystems in the document.

\section{PRESENTATION OF ONTOLOGY AS A DIAGRAM}

Checking the completeness and correctness of the established Database is carried out by visualization of ontology, namely, to create relationships and instances represented graphically by CNL-diagram (Fig.e 4). One skilled in the subject area will be easier to assess the correctness of the prepared guidance by imaging in CNLdiagram terms and relations between them [2].

The CNL-diagram help us to visualize in a more accessible way all the information, so in this way is more simple to understand the relation of every object, for example, (Figure 4) this diagram shows how the airplane has a direct relation to the engine, then how are different sub categories of type of engines according of the form of energy it accepts in order to create motion, and the type of motion it outputs, then each one 
of this type of engines have a sub-category, like reciprocatingengine have different position of the pistons or in case the rotor, then we can categories it in makes (brands), then models and each model have his own respective characteristics.

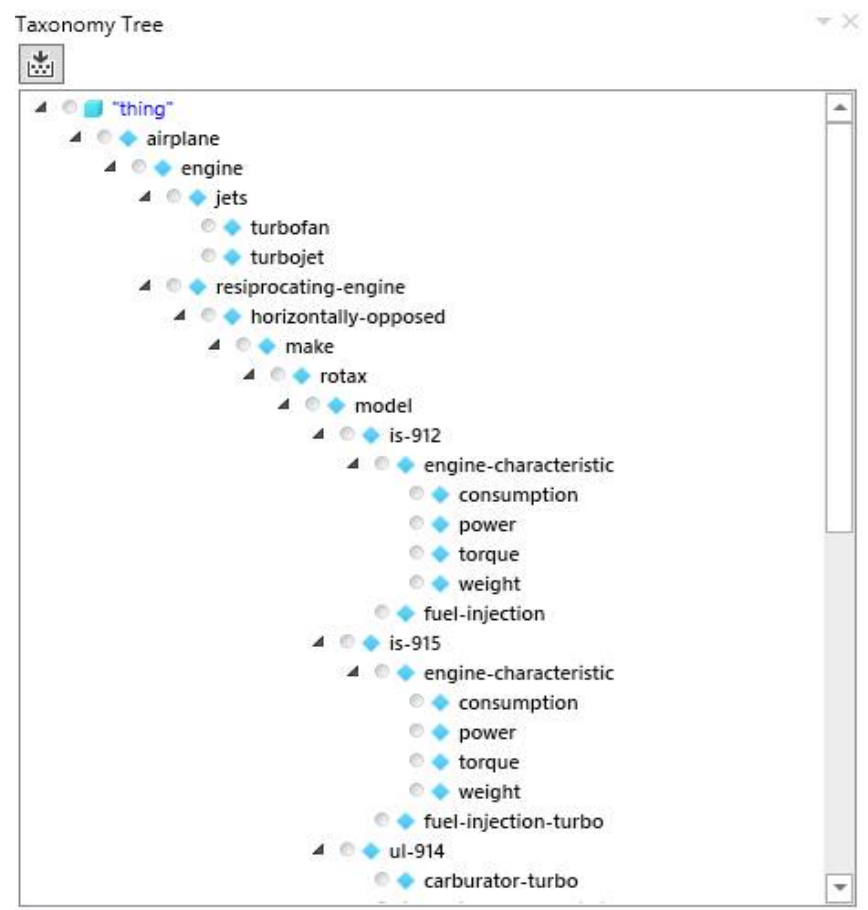

Taxonomy Tree Annotations

Fig. 3. Fragment of the Taxonomy Tree

\section{FORMALIZATION OF DESIGN}

The concept of formalization of design includes the description of the object and the process of its design with the help of graphic language, numbers, letters, codes and other symbols, that is, a combination of an ideographic set of functions of design solutions and technological functions, etc.

Therefore, to describe the detailed design, the degree of conformity of signs, the set of symbols and designations, and also the design and technological functions by which this description is carried out. That is, the structure of the aircraft, its shape, and dimensions can be described, for example, by means of functions or a finite number of such symbols, which are called parameters. The properties of the design of the aircraft or its subsystems (aggregates, finished products, etc.) can be described by another set of symbols, called characteristics,

Fluent Editor as a tool of information, supports the designer to work out and understand statistics in a faster and easiest way, making the task of formalization of a new design in a way more simple and user friendly.

\section{RELATED WORK}

There has been extensive work on the benefits of applying semantic technologies for the efficiency of model driven systems engineering, which has been the motivational background for this ontology development project.

For example, in the development of the aircraft ontology following the NEON process model. In particular, is described

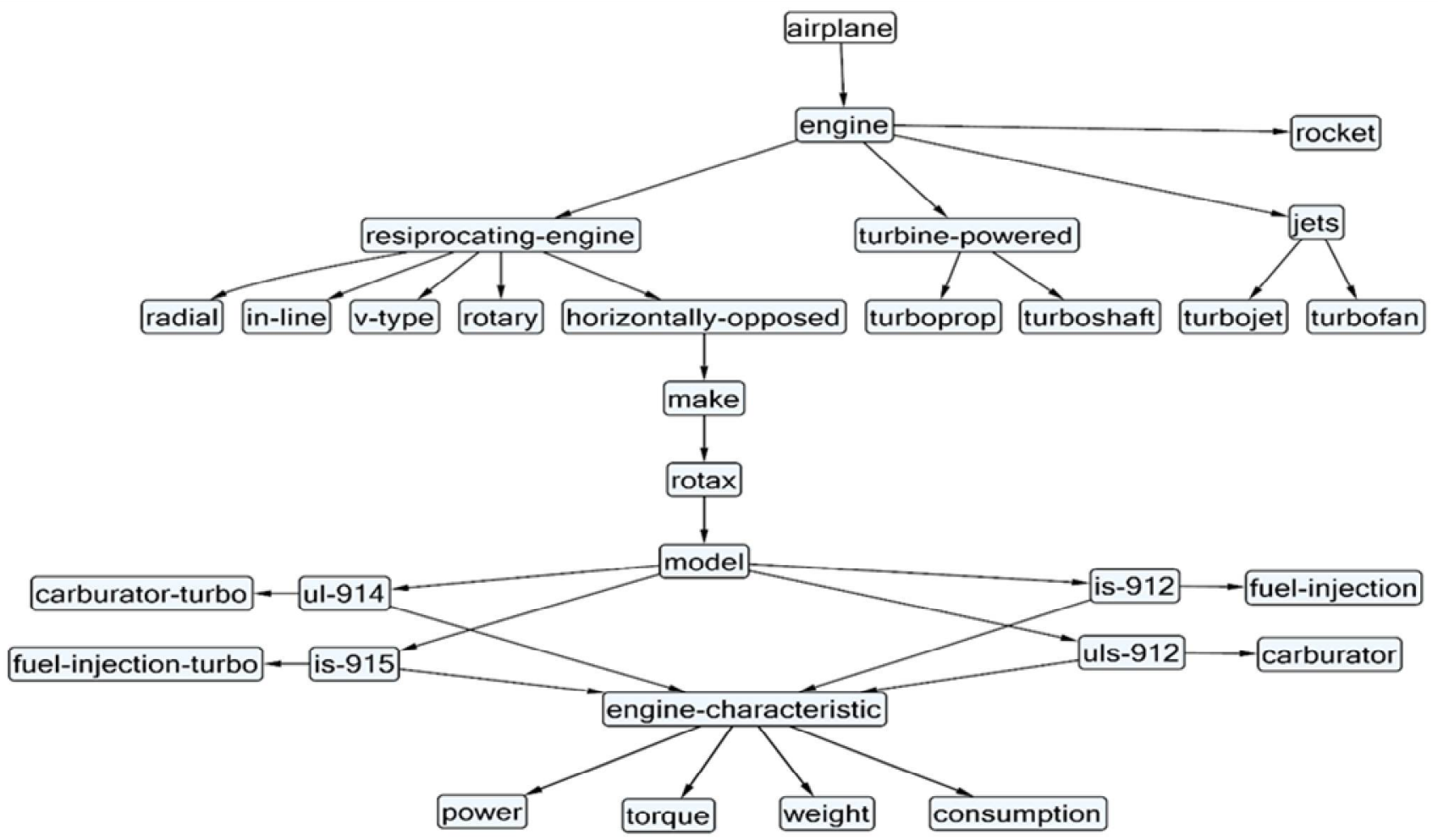

Fig. 4. CNL-diagram 
the experiences from applying the NEON methodology and the resulting aircraft ontology. The aircraft ontology is an OWL ontology that covers system decomposition and component parameters of a single aisle civil transport aircraft. It can be used as a common semantic reference during model comparison and transformation [6].

Another application that would work well together with this type of ontology is the Robot Designer actually in development in Samara University, this investigated the possibility of applying methods and techniques of artificial intelligence for accelerated training of student for preliminary design of the aircraft. It is aimed to help in the process of preliminary design of an aircraft [7].

Representing design alternatives as configurations of portbased objects is useful at the conceptual design stage when the geometry and spatial layout is still ill-defined. During the design process, as the designer makes additional decisions about the components and their interactions, these initial placeholders will be gradually transformed into specific port definitions. In terms of the port ontology, the incremental decisions of the designer will result in the addition of attributes to the port definitions, the sub-classing or refinement of attributes, or the addition of constraints on the attribute values [8].

\section{CONCLUSION}

"Fluent Editor" has the necessary mechanisms for the implementation, conceptualization and formalization of ontology. This program facilitates the use of ontologies for classification of objects and to visualize them so the student or researcher, could do an easiest and faster conclusions on themes related to the design area.

Classification of information on an easy way, effectively and fast, then working with it, will help in the future to cut times of design and research, so this will have a positive effect on the cost of development of new technology.

Something complicated like an airplane could be designed in some weeks or months, in comparison of the past that were months and years of exhaustive research for making reality the projects.

There is a project developed for the same creators of Fluent Editor, ADA (Ask Data Anything) this program allows exploring data by using natural language directly, this proves that in the future more complicated problems will be easily solved with support of semantic technologies.

\section{REFERENCES}

[1] Ch.M. Wijewickrema. Impact of an Ontology for Automatic Text Classifications, Annals of Library and Information Studies. Vol. 61, $\begin{array}{llll}\text { December } & \text { 2014, } & \text { 263-272. }\end{array}$ http://nopr.niscair.res.in/handle/123456789/30334.

[2] A.A. Orlova, N.M. Borgest. Text verification instruction manual using the ontologies / Proceedings of the 2016 Conference on Information Technologies in Science, Management, Social Sphere and Medicine (ITSMSSM 2016). ACSR - Advances in Comptuer Science Research. Vol. 51. 2016, pp.238-241.

[3] OWL - https://www.w3.org/TR/owl-semantics/

[4] FluentEditor 2014 - http://www.cognitum.eu/semantics /Fluent Editor/

[5] N.M. Borgest, M.D. Korovin. Formalization of design procedures in the engineer's educational strategy / Proceedings of the 2016 Conference on Information Technologies in Science, Management, Social Sphere and Medicine (ITSMSSM 2016). ACSR - Advances in Comptuer Science Research. Vol. 51. 2016, pp.524-527.

[6] M. Ast, Martin Glas, Tobias Roehm, Creating an Ontology for Airraft Design, Deutscher Luft- und Raumfahrtkongress 2013. DocumentID: 301356. - $11 \mathrm{p}$.

[7] N.M. Borgest, S.A. Vlasov, Al.A. Gromov, An.A. Gromov, M.D. Korovin, D.V. Shustova, Robot-designer: on the road to reality. Ontology of Designing, 5(4): 429-449, 2015. DOI: 10.18287/2223-95372015-5-4-429-449.

[8] Vei-Chung Liang, Christiaan J.J. Paredis. A port ontology for conceptual design of systems. Journal of Computing and Information science in engineering. Vol.4, September, 2004, pp.206-217. - DOI: 10.1115/1.17781 